\title{
Soluções otimizadas via Algoritmo Genético para o controle do mosquito transmissor da Dengue
}

\author{
Luiz G. Lyra, Helenice de O. F. Silva, Fernando L. P. dos Santos, \\ Daniela R. Cantane \\ Depto. de Bioestatística, IBB, UNESP, \\ 18618-970, Botucatu, SP \\ E-mail: \{lglyra,helenice,flpio,dcantane\}@ibb.unesp.br
}

\begin{abstract}
Resumo: Neste trabalho é apresentado estratégias otimizadas como propostas para o controle do mosquito transmissor da Dengue. Estas estratégias foram obtidas por meio do desenvolvimento do Algoritmo Genético ( $A G$ ) capaz de resolver o problema de otimização proposto. O modelo matemático baseia-se em Thomé, 2007, [4]. Este modelo descreve a dinâmica de mosquitos nas fases aquática e alada e considera variáveis de controles químicos, por inseticidas, e biológico, pela inserção de mosquitos machos estéreis no meio ambiente, [1, 3]. Soluções otimizadas para as variáveis de controle sugeridas pelo $A G$ proposto são apresentadas. $O$ AG aqui proposto mostrou ser uma ferramenta versátil e de grande aplicabilidade no controle das populações de mosquitos.
\end{abstract}

Palavras-chave: Aedes aegypti, controle ótimo, controle químico, controle biológico, mosquitos esteréis.

\section{Introdução}

A Dengue é uma doença febril causada pelo vírus do gênero Flavivírus da família Flaviviridae. A transmissão ocorre pela picada do mosquito fêmea, do gênero Aedes aegypti, contaminada por este vírus. Atualmente há quatro sorotipos: DEN-1, DEN-2, DEN-3 e DEN-4. Tal enfermidade é característica de regiões tropicais e subtropicais; Em particular, no Brasil, sua incidência tem crescido nas últimas décadas, [6]. O ciclo de vida do Aedes aegypti é compreendido por duas fases: aquática (ovo, larva e pupa) e alada (mosquito adulto). Devido à grande resistência dos ovos do Aedes às épocas secas, aliada às condições favoráveis ao seu desenvolvimento, sua erradicação ainda está longe de ser atingida. Assim, a única forma é estabelecer mecanismos de controle da doença que reduzam a população do mosquito transmissor. Atualmente, os mecanismos de controles existentes são: químico (uso de inseticidas), biológico (inserção de organismos vivos no meio ambiente) e mecânico (remoção de criadouros). O objetivo principal deste trabalho é obter estratégias otimizadas para o controle da Dengue. Para atingir este objetivo é proposto um AG que permite um amplo espaço de busca de soluções no problema e a inserção de valores de referências na resposta final e, de fácil implementação.

\section{Modelo Matemático}

Neste trabalho considera-se o modelo matemático desenvolvido e amplamente discutido por Thomé, $[4,5]$. Com base neste modelo, tem-se o seguinte problema de otimização: 
Minimizar $J=J\left[u_{1}, u_{2}\right]=\frac{1}{2} \int_{0}^{T}\left(c_{1} u_{1}^{2}+c_{2} u_{2}^{2}+c_{3} F^{2}-c_{4} S^{2}\right) d t$

sujeito a

$$
\begin{aligned}
& \frac{d A}{d t}=\phi\left(1-\frac{A}{C}\right) F-\left(\gamma+\mu_{A}\right) A \\
& \frac{d I}{d t}=r \gamma A-\left[\frac{\beta M}{M+S}+\frac{\beta_{S} S}{M+S}+\left(\mu_{I}+u_{1}\right)\right] I \\
& \frac{d F}{d t}=\frac{\beta M I}{M+S}-\left(\mu_{F}+u_{1}\right) F \\
& \frac{d M}{d t}=(1-r) \gamma A-\left(\mu_{M}+u_{1}\right) M \\
& \frac{d S}{d t}=u_{2}-\left(\mu_{S}+u_{1}\right) S \\
& R=\frac{\phi r \gamma \beta}{\left(\gamma+\mu_{A}\right)\left(\beta+\mu_{I}\right) \mu_{F}} \\
& A(0)=A_{0}=\frac{C(R-1)}{R} \\
& I(0)=I_{0}=\frac{r \gamma A_{0}}{\mu_{I}+\beta} \\
& F(0)=F_{0}=\frac{\left(\gamma+\mu_{A}\right) C A_{0}}{\phi\left(C-A_{0}\right)} \\
& M(0)=M_{0}=\frac{(1-r) \gamma A_{0}}{\mu_{M}} \\
& S(0)=S_{0}=0 \\
& u_{1} \geq 0 \\
& u_{2} \geq 0
\end{aligned}
$$

sendo $T$ é o período de uso do controle; $c_{1}, c_{2}, c_{3}$ e $c_{4}$ são as importâncias relativas do custo com inseticidas, da produção de mosquitos estéreis, do número de fêmeas fertilizadas e de preservação de mosquitos estéreis, respectivamente; $R=\frac{\phi r \beta}{\left(\gamma+\mu_{A}\right)\left(\beta+\mu_{I}\right) \mu_{F}}$ é a taxa de reprodutividade que mede o potencial máximo de reprodução de uma doença infecciosa; $R>1$ representa o equilíbrio endêmico estável. A variável de estado $U$, relacionada aos mosquitos fêmeas não-fertilizadas, é desacoplada do sistema dinâmico, (2) e dada por:

$$
\frac{d U}{d t}=\frac{\beta_{S} S I}{M+S}-\left(\mu_{U}+\mu_{1}\right) U
$$

As taxas de mortalidade per capita dos mosquitos são $\mu_{A}$ na fase aquática; $\mu_{I}$ para fêmeas imaturas; $\mu_{F}$ para fêmeas fertilizadas; $\mu_{U}$ para fêmeas não-fertilizada; $\mu_{M}$ para machos naturais; $\mu_{S}$ para machos estéreis. A taxa de oviposição da fêmea fertilizada $F$ é proporcional a sua densidade, depende do número de criadouros e é dada por $\phi\left(1-\frac{A}{C}\right)$, sendo $\phi$ a taxa de oviposição intrínseca; $C$ é a capacidade do meio relacionada com o número de nutrientes e espaço. Os mosquitos na fase aquática $A$ passam para a fase alada com uma taxa per capita $\gamma$, onde uma proporção $r$ são de fêmeas e $(1-r)$ são de machos. A mudança de fase das fêmeas imaturas $I$ para as fases fertilizadas $F$ e não-fertilizadas $U$ depende principalmente do número de encontros com os machos naturais $M$ e com os machos estéreis $S$ (irradiados). A probabilidade de encontro entre uma fêmea $I$ com um macho natural $M$ é dada por $\frac{M}{M+S}$. A taxa per capita com que as fêmeas são fertilizadas é dada por $\frac{\beta M}{M+S}$, sendo $\beta$ a taxa de acasalamento dos mosquitos naturais. A probabilidade de encontro de um macho estéril $S$ com uma fêmea $I$ não depende apenas do número de mosquitos machos irradiados e é dada por $\frac{p S}{M+S}$, em que $0 \leq p \leq 1$ é a proporção com que os mosquitos estéreis são colocados nos locais adequados. A taxa de acasalamento efetiva dos mosquitos estéreis é dada por $q \beta$, com $0 \leq q \leq 1$, sendo $q$ a redução do interesse em acasalamento do mosquito macho após o processo de irradiação. A taxa per capita com que as fêmeas $I$ são fertilizadas pelos mosquitos estéreis $S$ é dada por $\frac{\beta_{S} S}{M+S}$, em que $\beta_{S}=p q \beta$. 
Finalmente, $u_{2}$ é a taxa em que a população de mosquitos estéreis $S$ são colocadas no meio ambiente. A Figura 1 abaixo ilustra a dinâmica completa. Propõe-se a resolução do problema de controle ótimo mono-objetivo (1)-(2) e da Eq. (3) com uso de heurísticas. A Equação (1) representa a medida de desempenho do controle. Portanto, neste problema de controle ótimo, o objetivo é minimizar o valor do funcional $J$. Discute-se, assim, o AG proposto para resolução deste problema.

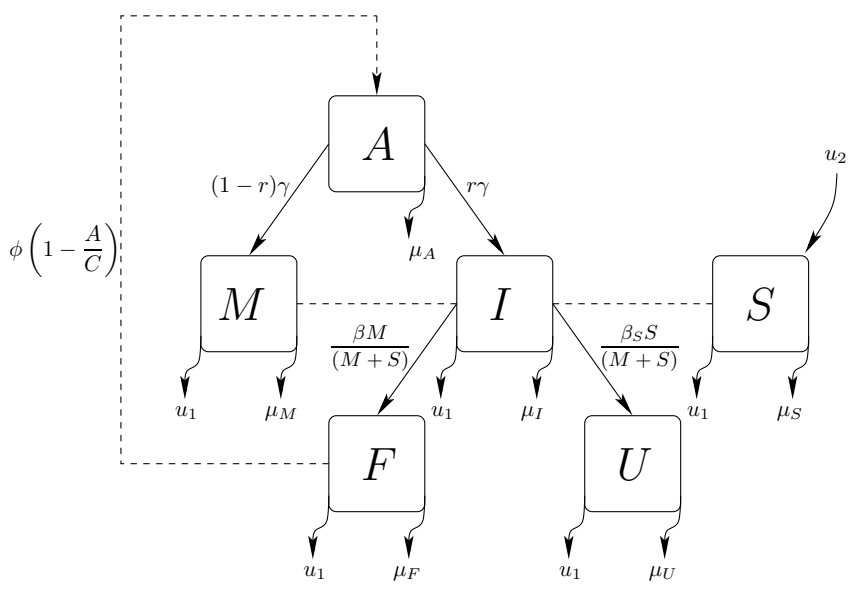

Figura 1: Diagrama da dinâmica populacional considerando as populações $A, I, F, M, S$ e $U$; $u_{1}$ e $u_{2}$ são os controles químico e biológico, respectivamente.

\section{Algoritmo Genético proposto}

Nesta seção apresentamos um Algoritmo Genético (AG) para resolução do problema de controle ótimo mono-objetivo (1)-(2), que consiste em determinar $\left(u_{1}^{*}, u_{2}^{*}\right)$ que minimiza $J\left[u_{1}, u_{2}\right]$ sujeito às restrições propostas, [2]. Os passos a seguir resumem o AG.

Passo 1 [Indivíduos]: definir a estrutura do indivíduo, ou cromossomo, $\left(u_{1}, u_{2}\right)$;

Passo 2 [População inicial]: gerar aleatoriamente uma populacao de $n$ indivíduos;

Passo 3 [Avaliação]: Calcular a aptidão de cada indivíduo da população, isto é, calcular a função fitness, $f_{i}$, para cada $u_{i}=\left(u_{1}, u_{2}\right)_{i}$ :

$$
f_{i}=\frac{1}{J\left[u_{i}\right] P_{i}}
$$

sendo $P_{i}= \begin{cases}1, & \text { se } u_{i} \text { é factível, } \\ 10^{5}, & \text { caso contrário. }\end{cases}$

$J\left[u_{i}\right]$ é o valor da função objetivo avaliada em $u_{i}$, obtido pelo método clássico de integração numérica 1/3 de Simpson Generalizada.

Passo 4 [Elite]: Armazenar os indivíduos de melhor aptidão no conjunto nomeado Elite;

Passo 5 [Seleção]: Selecionar ps\% de indivíduos da população e armazenar na população intermediária para o cruzamento (próximo passo);

Passo 6 [Crossover]: Fazer o cruzamento dois a dois entre os indivíduos selecionados no Passo 5;

Passo 7 [Mutação]: Selecionar com a probabilidade pm os indivíduos da população que sofrerão mutação; 
Passo 8 [Nova população]: Selecionar os $n$ indivíduos de melhor aptidão dentre a população intermediária e a população anterior;

Passo 9 [Avaliação]: Calcular a aptidão de cada indivíduo da população e atualizar o conjunto Elite;

Passo 10 [Critério de parada]: Se o critério de parada for satisfeito (número de gerações), vá para o Passo 11; Caso contrário, vá para o Passo 5.

Passo 11 [Fim]: A solução é o melhor indivíduo $u_{i}$ presente no conjunto Elite.

Dessa forma, as propriedades deste algoritmo são: $(i)$ fácil de ser implementado; (ii) permite inserir novas restrições ao problema, sem dificultar sua resolução. Assim, pode-se inserir valores de referências sobre as saídas dos estados $(A, I, F, M, S$ e $U)$ do sistema (2), satisfazendo $(i)$ e $(i i)$. Considerando o estado $F$, seja $F_{\text {fixo }}$ o tamanho máximo de fêmeas fertilizadas para um dado tempo $t_{\text {fixo }}$. Com isso, $F(t) \leq F_{\text {fixo }}$, para todo $t \geq t_{\text {fixo }}$, é uma restrição fundamental no processo de controle. Como consequência imediata, tem-se que o crescimento desta população será, assim, controlado. A seção seguinte apresenta os resultados obtidos por meio do AG proposto neste trabalho.

\section{Resultados}

A Tabela 1 apresenta os parâmetros utilizados no problema de otimização (1)-(2). Utilizando estes parâmetros, obtêm-se as condições iniciais apresentadas na Tabela 2. Estas condições traduzem o equilíbrio do sistema e pior situação da doença para $R>1$. Assim, o controle é aplicado quando a população de mosquitos é densa. As Tabelas 3 e 4 mostram, respectivamente, o valor para a restrição sobre $F$ e os parâmetros utilizados no AG. Na Tabela 4, $T$ é o período de uso do controle; $G$ é o número de gerações; $n$ é o número de indivíduos da população; $P$ é a penalização imposta aos indivíduos infactíveis; $K$ é o número de elementos da elite; $p s \%$ é a porcentagem de indivíduos selecionados para o crossover e $p m$ é a probabilidade de um indivíduo sofrer mutação.

Tabela 1: Parâmetros utilizados no modelo, [4].

\begin{tabular}{cccccccccc}
\hline$C$ & $\gamma$ & $\phi$ & $r$ & $\beta$ & $\mu_{A}$ & $\mu_{I}$ & $\mu_{F}$ & $\mu_{U}$ & $\mu_{M}$ \\
\hline 13 & 0,07 & 0,5 & 0,5 & 1 & 0,05 & 0,05 & 0,05 & 0,05 & 0,1 \\
\hline
\end{tabular}

Tabela 2: Condiç̃es iniciais utilizadas, [4].

\begin{tabular}{ccccc}
\hline $\mathrm{A}(0)$ & $\mathrm{I}(0)$ & $\mathrm{F}(0)$ & $\mathrm{M}(0)$ & $\mathrm{S}(0)$ \\
\hline 8,3200 & 0,2773 & 5,5467 & 2,9120 & 0 \\
\hline
\end{tabular}

Tabela 3: Parâmetros para a restrição em $F$.

\begin{tabular}{cc}
\hline$t_{\text {fixo }}$ & $F_{\text {fixo }}$ \\
\hline 30 & 0,3 \\
\hline
\end{tabular}

Tabela 4: Parâmetros utilizados no Algoritmo Genético.

\begin{tabular}{ccccccc}
\hline$T$ & $G$ & $n$ & $P$ & $K$ & $p s \%$ & $p m$ \\
\hline 120 & 1000 & 500 & $10^{5}$ & 50 & $80 \%$ & 0,05 \\
\hline
\end{tabular}

Tabela 5: Parâmetros utilizados nos coeficientes $c_{i}, i=1, \ldots, 4$.

\begin{tabular}{cccc}
\hline$c_{1}$ & $c_{2}$ & $c_{3}$ & $c_{4}$ \\
\hline 1 & 1 & 1 & 1 \\
\hline
\end{tabular}


Com base em [2], normaliza-se as parcelas do funcional $J$, tal que tenham a mesma magnitude. Os coeficientes $c_{i}, i=1, \ldots, 4$, são dados conforme a Tabela 5. As equações diferencias que descrevem a dinâmica das populações de mosquitos é resolvido aplicando-se o método de Runge-Kutta de quarta ordem. O problema de controle ótimo, bem como o AG proposto foi implementado em linguagem C. Para esta implementação foi utilizado o período de tempo $T$ igual a 120 dias. Para avaliar a aplicabilidade e a versatilidade do AG em sugerir estratégias otimizadas de controle de mosquitos, elaborou-se as situações 1 e 2 abaixo.

Situação 1: $u_{1}(t)$ e $u_{2}(t)$ constantes em períodos de aplicação de 10 em 10 dias ao longo do tempo $T$.

As Figuras 2 e 3 mostram as variáveis de controle $u_{1}(t)$ e $u_{2}(t)$, respectivamente, sugeridas de forma otimizada pelo AG aqui proposto. Estes valores são então utilizados na resolução do sistema (2). As Figuras 4 e 5 mostram os efeitos desses controles na dinâmica da população de mosquitos fêmeas. Em particular, pode-se notar o decrescimento da população de fêmeas fertilizadas, Figura 4.

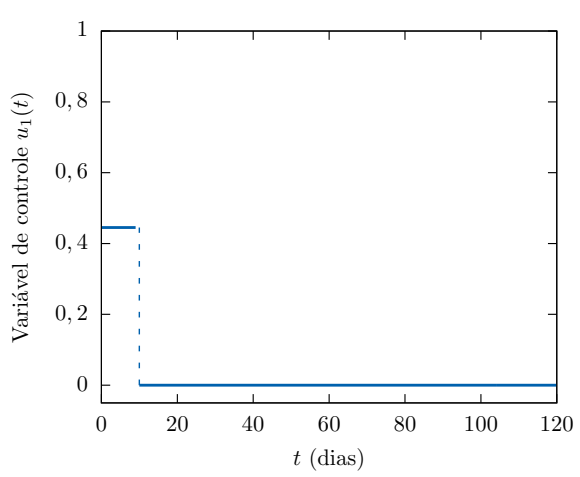

Figura 2: Variável de controle $u_{1}(t)$ referente Figura 3: Variável de controle $u_{2}(t)$ referente ao investimento com inseticida.

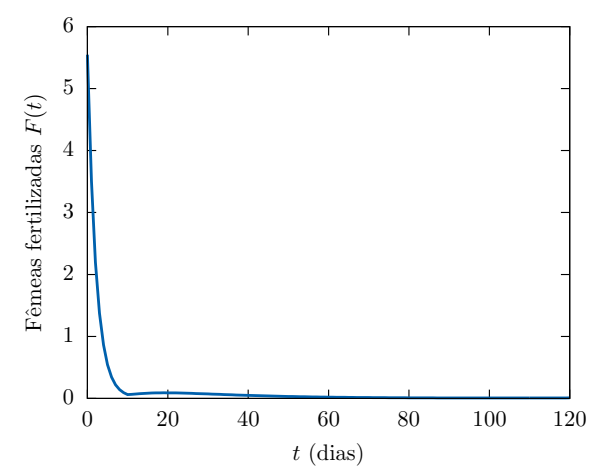

Figura 4: População de mosquitos fêmeas fer- Figura 5: População de mosquitos fêmeas nãotilizadas $F$.

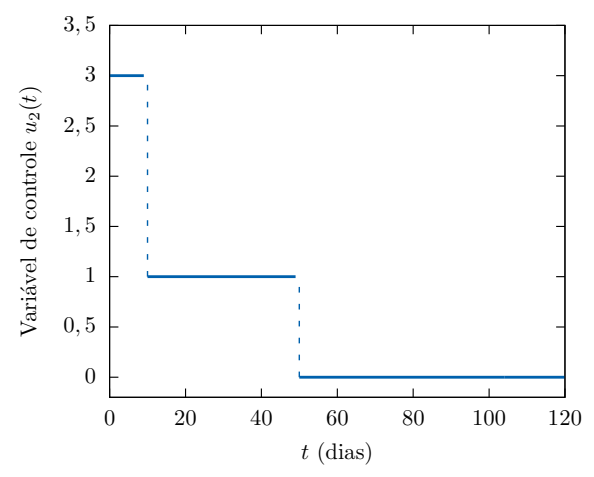
ao investimento com mosquitos machos estéreis.

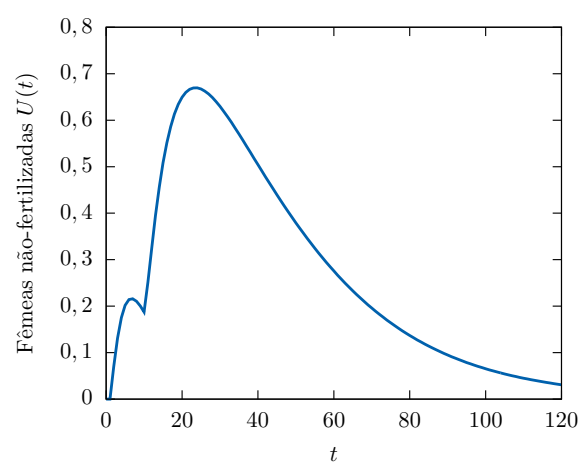

fertilizadas $U$.

Situação 2: Iniciar com $u_{1}(t)$ e $u_{2}(t)$ constantes. Neste caso, o AG determina a melhor forma de aplicação.

Nesta situação, o AG sugere a quantidade que se deve aplicar de cada controle e o melhor período de aplicação. Aqui, AG aponta que quando o investimento de um dos controles é alto, o outro deve ser baixo. Este comportamento pode ser visto nas Figuras 6 e 7 que apontam altos valores para $u_{2}(t)$ e baixos valores para $u_{1}(t)$, no período entre 30 e 120 dias. Isto resulta em 
baixos investimentos na aplicação desses controles e mostra a versatilidade do AG aqui proposto. As Figuras 8 e 9 mostram os efeitos desses controles na dinâmica da população de mosquitos fêmeas. Novamente, é possível notar o decrescimento da população de fêmeas fertilizadas, Figura 8.

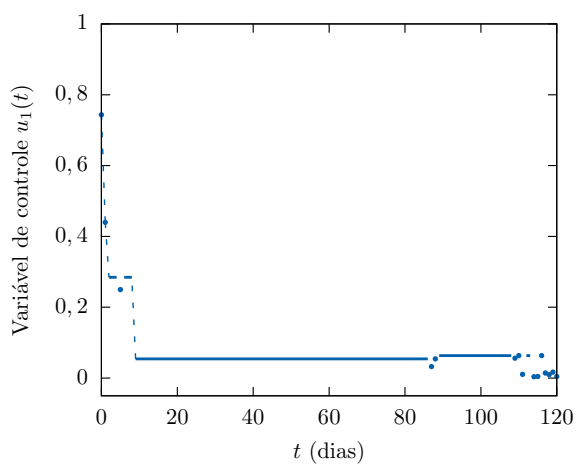

Figura 6: Variável de controle $u_{1}(t)$ referente ao investimento com inseticida.

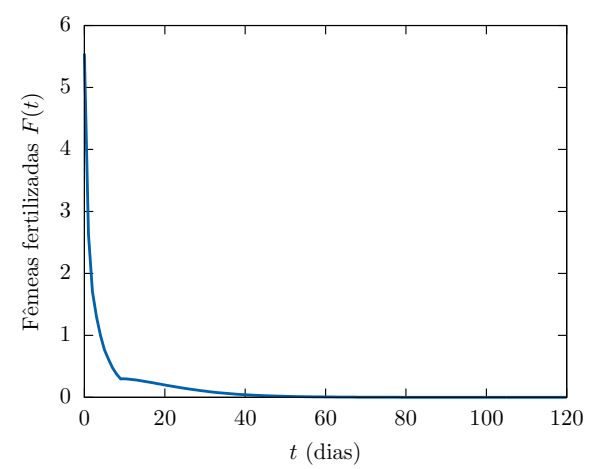

Figura 8: População de mosquitos fêmeas fer- Figura 9: População de mosquitos fêmeas nãotilizadas $F$.

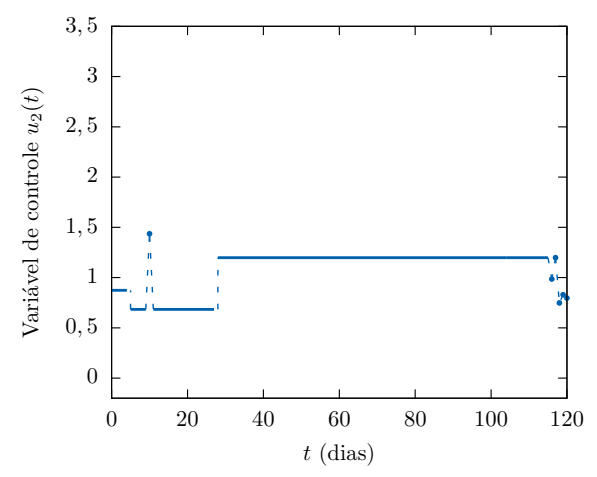

Figura 7: Variável de controle $u_{2}(t)$ referente ao investimento com mosquitos machos estéreis.

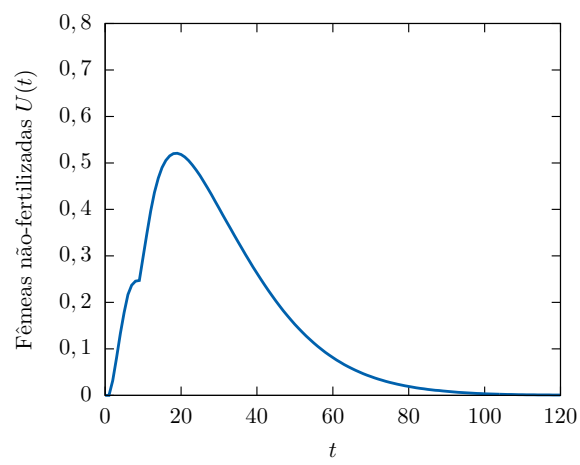
fertilizadas $U$.

A Tabela 6 apresenta os valores do funcional $J$ para as situações 1 e 2 . Como o objetivo é minimizar o valor de $J$, segue que a situação $2(J=1,96)$ representa, dentre as duas, a melhor estratégia que otimizará a aplicação dos controles ao longo do tempo considerado.

Tabela 6: Comparação dos valores de $J$.

\begin{tabular}{cc}
\hline Situação & $J$ \\
\hline 1 & 2,57 \\
2 & 1,96 \\
\hline
\end{tabular}

\section{Conclusões}

Como conclusões acerca deste trabalho, cujo objetivo foi a obtenção de estratégias otimizadas para o controle da Dengue via método heurístico, tem-se que o AG aqui proposto:

- mostrou ser uma ferramenta versátil e de grande aplicabilidade na obtenção de resultados otimizados para o problema em questão;

- possui as propriedades: (i) fácil de ser implementado; (ii) vantagem de inserir novas restrições ao problema, sem impor novas dificultades na resolução; 
- com o Passo 3, permitiu impor penalidades sobre as soluções infactíveis, ampliando o espaço de busca na região de factibilidade;

- sugeriu como melhor estratégia de controle, dentre as situações analisadas, aquela em que as variações dos controles e os períodos de aplicações são ambos determinados pelo algoritmo.

\section{Referências}

[1] A. C. Bartlett; R. T. Staten, The sterile release method and other genetic control strategies, Radcliffe's IPM World Textbook, University of Minesota, 1996.

[2] Kalyanmoy Deb and Deb Kalyanmoy. Multi-Objective Optimization Using Evolutionary Algorithms. John Wiley \& Sons, Inc., New York, NY, USA, 2001.

[3] L. Esteva \& H. M. Yang: Mathematical Model to Assess the Control of Aedes aegypti Mosquitoes by Sterile Insect Technique, Mathematical Biosciences, 198: 132-147 (2005).

[4] R. C. A. Thomé. Controle ótimo aplicado na estratégia de combate ao Aedes aegypti utilizando inseticida e mosquitos estéreis, Tese de Doutorado, IMECC/UNICAMP, 2007.

[5] R. C. A. Thomé; H. M. Yang, L. Esteva. Optimal control of Aedes aegypti mosquitoes by the sterile insect technique and insecticide, Mathematical Biosciences, Elsevier, 223 (2010), 12-23.

[6] World Health Organization. Dengue: Guidelines for Diagnosis, Treatment, prevention and control, 2nd edn. Geneva: WHO, 2009. 